\title{
OCTOBRE 1972
}

54. ANNÉE - N•646

\section{revue internationale de la croix-rouge}

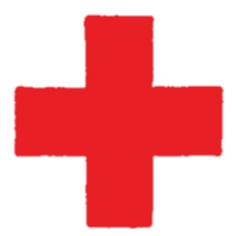

INTER ARMA CARITAS

GENEVE

COMITE INTERNATIONAL DE LA CROIX-ROUGE FONDÉ EN 1863. 


\section{COMITÉ INTERNATIONAL DE LA CROIX-ROUGE}

MM. MARCEL A. NAVILLE, président (membre depuis 1967)

JEAN PICTET, docteur en droit, président de la Commission juridique, vice-president (1967)

HARALD HUBER, docteur en droit, juge fédéral, vice-président (1969)

PAUL RUEGGER, ambassadeur, président du CICR de 1948 à 1955 (1948)

GUILLAUME BORDIER, ingénieur dipl. E.P.F., M.B.A. Harvard, banquier (1955)

HANS BACHMANN, docteur en droit, Stadtrat de Winterthour (1958)

JACQUES FREYMOND, docteur ès lettres, directeur de l'Institut universitaire des hautes études internationales, professeur à l'Université de Genève (1959)

DIETRICH SCHINDLER, docteur en droit, professeur à l'Université de Zurich (1961)

M $^{116}$ MARJORIE DUVILLARD, infirmière (1961)

MM. MAX PETITPIERRE, docteur en droit, ancien président de la Confédération suisse (1961)

ADOLPHE GRAEDEL, député au Conseil national suisse de 1951 à 1963, ancien secrétaire général de la Fédération internationale des ouvriers sur métaux (1965)

$M^{m}{ }^{m}$ DENISE BINDSCHEDLER-ROBERT, docteur en droit, professeur à l'Institut universitaire des hautes études internationales (1967)

MM. JACQUES F. DE ROUGEMONT, docteur en médecine (1967)

ROGER GALLOPIN, docteur en droit, ancien directeur général (1967)

WALDEMAR JUCKER, docteur en droit, secrétaire de l'Union syndicale suisse (1967)

VICTOR H. UMBRICHT, docteur en droit, administrateur (1970), en congé

PIERRE MICHELI (1971)

Membres honoraires: M. JACQUES CHENEVIĖRE, vice-président d'honneur; $\mathrm{M}^{\mathrm{H} \boldsymbol{H}}$ LUCIE ODIER, vice-présidente d'honneur; MM. CARL J. BURCKHARDT, PAUL CARRY, $M^{\text {me }}$ MARGUERITE GAUTIER-VAN BERCHEM, MM. SAMUEL A. GONARD, EDOUARD

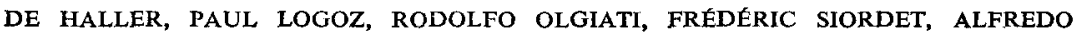
VANNOTTI, ADOLF VISCHER.

Direction: MM. JEAN-LOUIS LE FORT, secrétaire général;

CLAUDE PILLOUD, directeur du département des principes et du droit;

JEAN-PIERRE MAUNOIR, sous-directeur, chargé ad interim de la direction du département des operations. 


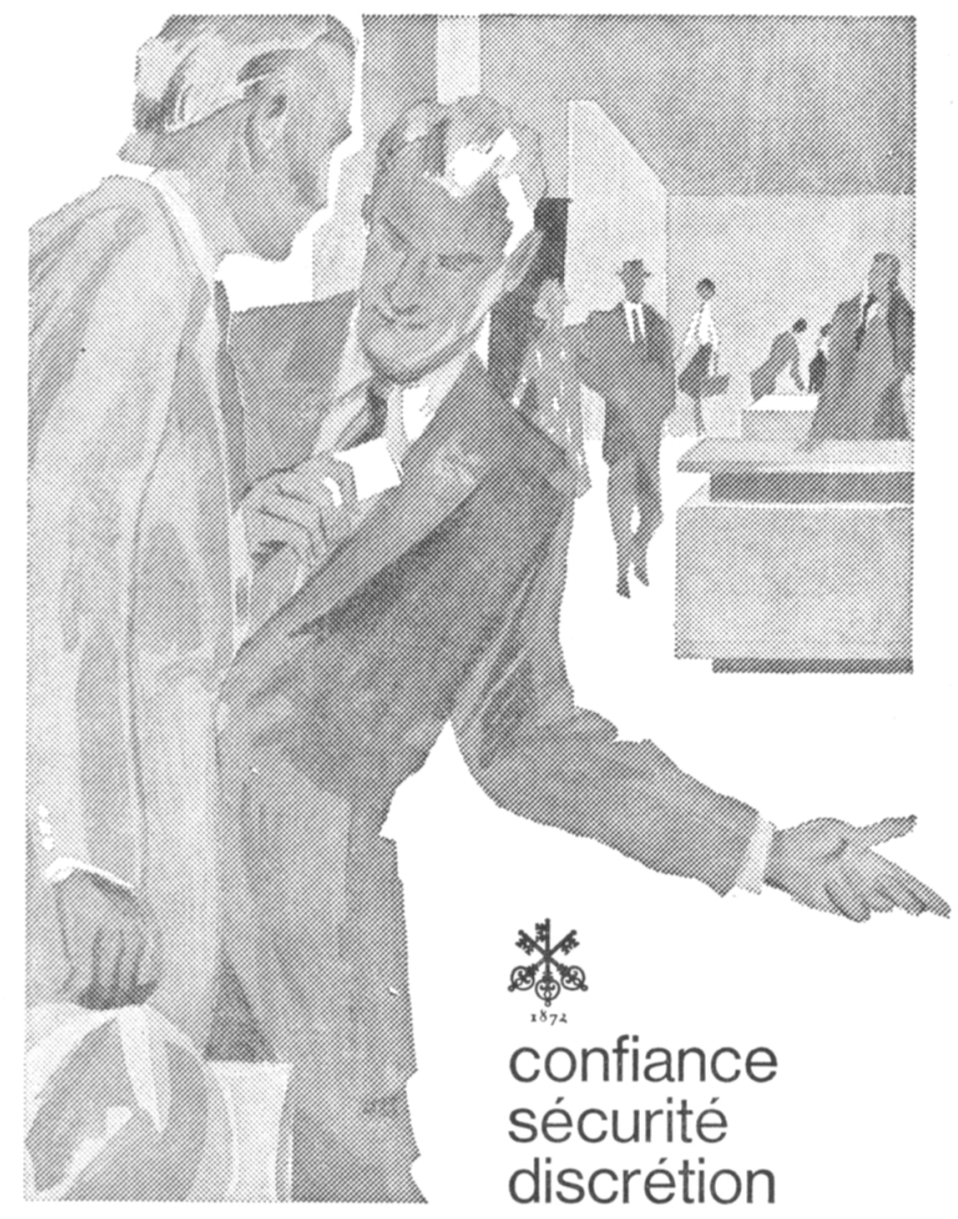

\section{SOCIÉTÉ DE BANQUE SUISSE SWISS BANK CORPORATION}

Siège de Genève: 2, rue de la Confédération Tél. 266211 


\section{SOCIETE FIDUCIAIRE ROMANDE OFOR S.A.}

Place Saint-Gervais 1, Genève - Tél. 317050

- Expertises, revisions et organisations comptables

$\downarrow$ Domiciliation et administration de sociétés

$\downarrow$ Assainissements et liquidations

— Interventions et conseils en matière fiscale

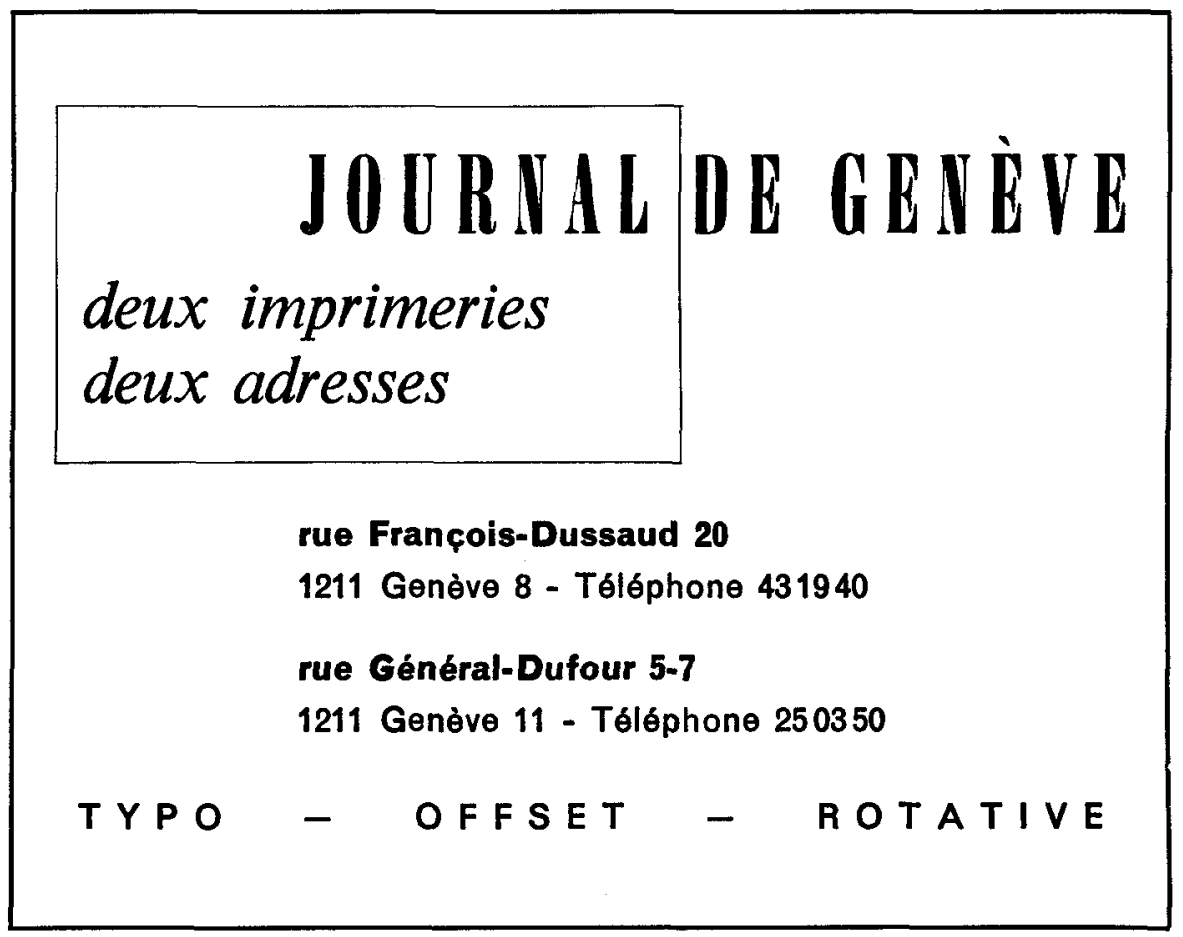




\section{REVUE INTERNATIONALE \\ DE LA CROIX-ROUGE}

SOMMAIRE

COMITÉ INTERNATIONAL DE LA CROIX-ROUGE

DANS LE MONDE DE LA CROIX-ROUGE

FAITS ET DOCUMENTS

\section{OCTOBRE 1972 - N646}

Paul Weis : Les réfugiés et les droits de l'homme (I) 595

Manfred Müller : Henry Dunant et Rudolf Müller 606

Reconnaissance de la Société du Croissant-Rouge de Bahrein (487e circulaire aux Comités centraux) . . . . . . . . . . . . . . 620

Le Comité international de la Croix-Rouge et la prise d'otages

Activités extérieures:

Sous-continent asiatique - République khmère

- République du Vietnam - Moyen-Orient

- Argentine - Jamaïque . . . . . . . . .

A Genève :

Le Président de la République fédérale d'Allemagne au CICR . . . . . . . . . . . . . . 629

Réunion d'information des Sociétés nationales 629

Agence centrale de recherches . . . . . . 631

Le « Manuel du soldat» . . . . . . . . . . 633

Commission permanente de la Croix-Rouge internationale ............. 636

Conférence européenne de la Croix-Rouge de la Jeunesse . . . . . . . . . . . . . . . . . 637

La Croix-Rouge et le don gratuit du sang . . . . 641

International Society for the Study of Comparative Public Law. . . . . . . . . . . . . 642

Réunion d'un groupe d'experts sur le napalm et les autres armes incendiaires . . . . . . 644

Congrès d'Amnesty International . . . . . 646 


\section{INTERNATIONAL REVIEW \\ OF THE RED CROSS}

\section{BUPPLÉMENTS \\ DE LA REVUE}

EN LANGUE ESPAGNOLE

EN LANGUE ALLEMANDE

\section{REVUE INTERNATIONALE DE LA CROIX-ROUGE}

Une édition en langue anglaise paraît chaque mois. Elle est en principe identique à l'édition française, et peut être obtenue aux mêmes conditions.

Sachiko Hashimoto: La Cruz Roja y la educación de la juventud - Reconocimiento de la Sociedad de la Media Luna Roja de Bahrein (Circular núm. 487 a los Comités Centrales) - El Comité Internacional de la Cruz Roja y la toma de rehenes - Servicio de Radiocomunicaciones del CICR - Publicación de un informe oficial.

Manfred Müller: Henry Dunant und Rudolf Müller Anerkennung des Roten Halbmonds von Bahrein (487. Rundschreiben an die Zentralkomitees) - Das Internationale Komitee vom Roten Kreuz und die Festnahme von Geiseln - Der Präsident der Bundesrepublik Deutschland beim IKRK - Informationstagung der nationalen Gesellschaften.

Elle est publiée chaque mois par le Comité international de la Croix-Rouge.

7, avenue de la Paix, 1211 Genève 1 (Suisse) - Compte de chèques postaux $12-1767$.

Abonnement un an: Fr. 30,--; le numéro: Fr. 3,--

RÉDACTION : J.-G. LOSSIER

Seuls les textes signés par le Comité international de la Croix-Rouge engagent la responsabilité de celui-ci. 